\title{
OBSTETRICS
}

\section{Defining failed induction of labor}

William A. Grobman, MD, MBA; Jennifer Bailit, MD, MPH; Yinglei Lai, PhD; Uma M. Reddy, MD, MPH; Ronald J. Wapner, MD; Michael W. Varner, MD; John M. Thorp Jr, MD; Kenneth J. Leveno, MD; Steve N. Caritis, MD; Mona Prasad, DO; Alan T. N. Tita, MD, PhD; George Saade, MD; Yoram Sorokin, MD; Dwight J. Rouse, MD; Sean C. Blackwell, MD; Jorge E. Tolosa, MD, MSCE; for the Eunice Kennedy Shriver National Institute of Child Health and Human Development Maternal-Fetal Medicine Units Network

BACKGROUND: While there are well-accepted standards for the diagnosis of arrested active-phase labor, the definition of a "failed" induction of labor remains less certain. One approach to diagnosing a failed induction is based on the duration of the latent phase. However, a standard for the minimum duration that the latent phase of a labor induction should continue, absent acute maternal or fetal indications for cesarean delivery, remains lacking.

OBJECTIVE: The objective of this study was to determine the frequency of adverse maternal and perinatal outcomes as a function of the duration of the latent phase among nulliparous women undergoing labor induction.

STUDY DESIGN: This study is based on data from an obstetric cohort of women delivering at 25 US hospitals from 2008 through 2011. Nulliparous women who had a term singleton gestation in the cephalic presentation were eligible for this analysis if they underwent a labor induction. Consistent with prior studies, the latent phase was determined to begin once cervical ripening had ended, oxytocin was initiated, and rupture of membranes had occurred, and was determined to end once $5-\mathrm{cm}$ dilation was achieved. The frequencies of cesarean delivery, as well as of adverse maternal (eg, postpartum hemorrhage, chorioamnionitis) and perinatal (eg, a composite frequency of seizures, sepsis, bone or nerve injury, encephalopathy, or death) outcomes, were compared as a function of the duration of the latent phase (analyzed with time both as a continuous measure and categorized in 3-hour increments).
RESULTS: A total of 10,677 women were available for analysis. In the vast majority (96.4\%) of women, the active phase had been reached by 15 hours. The longer the duration of a woman's latent phase, the greater her chance of ultimately undergoing a cesarean delivery $(P<.001$, for time both as a continuous and categorical independent variable), although $>40 \%$ of women whose latent phase lasted $\geq 18$ hours still had a vaginal delivery. Several maternal morbidities, such as postpartum hemorrhage $(P<.001)$ and chorioamnionitis $(P<.001)$, increased in frequency as the length of latent phase increased. Conversely, the frequencies of most adverse perinatal outcomes were statistically stable over time.

CONCLUSION: The large majority of women undergoing labor induction will have entered the active phase by 15 hours after oxytocin has started and rupture of membranes has occurred. Maternal adverse outcomes become statistically more frequent with greater time in the latent phase, although the absolute increase in frequency is relatively small. These data suggest that cesarean delivery should not be undertaken during the latent phase prior to at least 15 hours after oxytocin and rupture of membranes have occurred. The decision to continue labor beyond this point should be individualized, and may take into account factors such as other evidence of labor progress.

Key words: labor induction, latent phase, outcomes

\section{Introduction}

Induction of labor has become an increasingly utilized obstetric intervention. Over the last 2 decades, its use has more than doubled, and at present, approximately 1 in 4 pregnant women have their labor induced. ${ }^{1}$ One conundrum faced by clinicians who are caring for women undergoing labor induction is whether the benefits outweigh the risks of continuing labor when a woman remains in the latent phase for an extended period of time. When a

Cite this article as: Grobman WA, Bailit J, Lai Y, et al. Defining failed induction of labor. Am J Obstet Gynecol 2018;218:122.e1-8.

0002-9378/free

(C) 2017 Elsevier Inc. All rights reserved.

https://doi.org/10.1016/j.ajog.2017.11.556

\section{EDITORS' CHOICE}

cesarean delivery occurs in the latent phase of a labor induction, the indication is sometimes labeled as "failed." However, there has not been consensus regarding the criterion for this indication, and as a result, the approach to obstetric management in the latent phase for women undergoing labor induction varies among providers and institutions. ${ }^{2}$

Rouse et $\mathrm{al}^{3}$ formulated one approach to defining a failed induction. ${ }^{3}$ They defined the latent phase as beginning when both oxytocin had been initiated and rupture of membranes (ROM) had occurred, and ending at either $4-\mathrm{cm}$ dilation and $90 \%$ effacement or $5-\mathrm{cm}$ dilation regardless of effacement.
Obstetric outcomes were then studied as a function of the length of the latent phase in induced labors. They concluded that the latent phase could be allowed to extend to at least 12 hours without excess obstetric morbidity. However, their study population was relatively small and from a single site, and they could not adequately assess durations of the latent phase $>12$ hours. Three other studies were performed that approached the diagnosis of a failed induction from this perspective, and to varying degrees had similar methodological limitations. ${ }^{4-6}$

Determining a standard and evidencebased criterion for a cesarean that is performed in the latent phase for the sole reason that the patient has not entered the active phase is important if unnecessary cesarean deliveries are to be 
FIGURE

Flowchart illustrating composition of study population

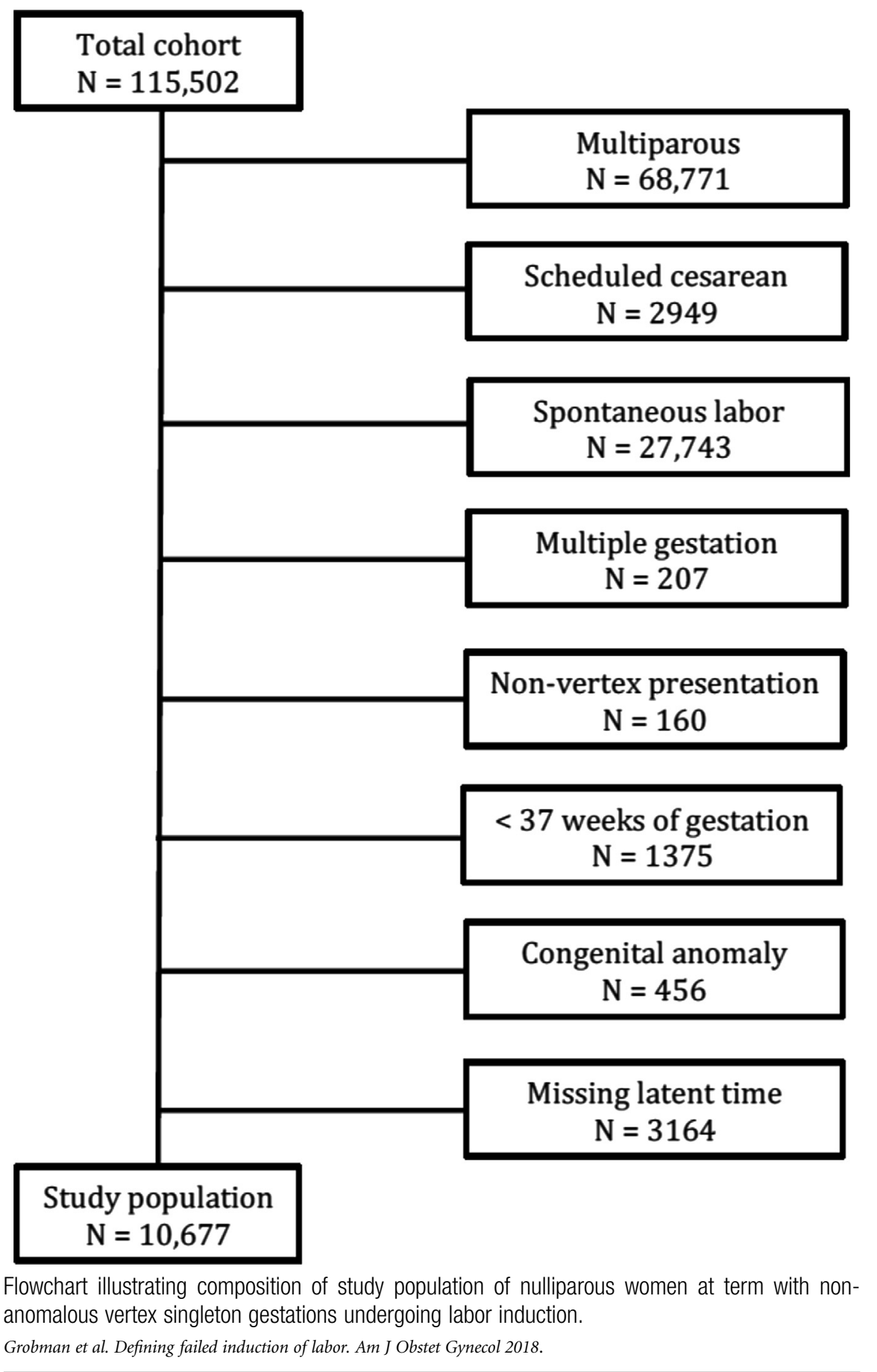

minimized and interinstitutional comparisons of care are to be possible. ${ }^{7}$ Thus, the purpose of this analysis was to determine, among a large and geographically varied population of nulliparous women undergoing labor induction, the maternal and neonatal outcomes associated with the length of the latent phase of labor.

\section{Materials and Methods}

From 2008 through 2011, investigators at the Eunice Kennedy Shriver National Institute of Child Health and Human
Development Maternal-Fetal Medicine Units Network performed an observational study (ie, the APEX study). In this study, patient characteristics, intrapartum events, and pregnancy outcomes were collected on all women of at least 23 $0 / 7$ weeks with a live fetus on admission and delivered on randomly selected days representing one third of deliveries over a 3-year period at 25 participating hospitals. Trained and certified research personnel abstracted all charts. All centers obtained institutional review board approval and a waiver of informed consent. Full details of the technique of data collection were described previously. ${ }^{8}$

Women were considered eligible for this analysis if they were nulliparous; had a singleton, cephalic gestation at $\geq 37$ weeks; and underwent labor induction. The duration of the latent phase was defined in a similar fashion to that first elaborated by Rouse et $\mathrm{al}^{3}$ and subsequently used by others in their analyses of the latent phase during labor induction. Specifically, the latent phase of labor in the setting of induction was defined to begin once any cervical ripening had been completed (ie, when it was no longer used), oxytocin had begun, and ROM (either spontaneously or artificially) had occurred. Latent phase labor was defined to end once at least $5-\mathrm{cm}$ dilation had been reached (or if cesarean occurred before that dilation). Women were excluded from the primary analysis if any of the times needed to calculate the length of the latent phase (eg, time at ROM, time at oxytocin initiation, time at least $5 \mathrm{~cm}$ was reached) were not available in the chart and, correspondingly, the length of the latent phase could not be determined.

Patient outcomes, including the frequency of cesarean delivery, adverse maternal outcomes (clinically diagnosed chorioamnionitis, postpartum hemorrhage, hysterectomy), and adverse neonatal outcomes were compared as a function of the duration of the latent phase. The primary adverse neonatal outcome was a composite that was defined to occur when a neonate had any of the following: seizures, culture-proven 


\begin{tabular}{|c|c|}
\hline Characteristic & $\mathrm{N}=10,677$ \\
\hline Maternal age, y & $26.4 \pm 6.1$ \\
\hline Body mass index, $\mathrm{kg} / \mathrm{m}^{2}$ & $32.2 \pm 6.7$ \\
\hline Gestational age, wk & $39.8 \pm 1.3$ \\
\hline \multicolumn{2}{|l|}{ Race/ethnicity } \\
\hline White & $5724(53.6)$ \\
\hline Black & $2153(20.2)$ \\
\hline Hispanic & $1665(15.6)$ \\
\hline Asian & $570(5.3)$ \\
\hline Other & $565(5.3)$ \\
\hline \multicolumn{2}{|l|}{$\begin{array}{l}\text { Reason for labor } \\
\text { induction }\end{array}$} \\
\hline $\begin{array}{l}\text { Maternal medical } \\
\text { condition }^{\mathrm{a}}\end{array}$ & $2562(24.0)$ \\
\hline Late or postterm & $3004(28.1)$ \\
\hline Fetal status $^{b}$ & $1636(15.3)$ \\
\hline PROM & 1725 (16.2) \\
\hline Elective & $1468(13.8)$ \\
\hline Other & $282(2.6)$ \\
\hline Cervical ripening & $5582(52.3)$ \\
\hline Epidural use & $10,038(95.0)$ \\
\hline Birthweight, g & $3369 \pm 477$ \\
\hline \multicolumn{2}{|c|}{ Data presented as mean $\pm \mathrm{SD}$ or $\mathrm{N}(\%)$. } \\
\hline \multicolumn{2}{|c|}{ PROM, premature rupture of membranes. } \\
\hline \multicolumn{2}{|c|}{$\begin{array}{l}\text { a Includes maternal comorbidities, such as hypertensive } \\
\text { disease and diabetes mellitus, that are indications for } \\
\text { labor induction; }{ }^{b} \text { Includes fetal growth restriction, } \\
\text { oligohydramnios, nonreassuring antepartum } \\
\text { surveillance. }\end{array}$} \\
\hline \multicolumn{2}{|c|}{$\begin{array}{l}\text { Grobman et al. Defining failed induction of labor. } \\
\text { Am J Obstet Gynecol } 2018 \text {. }\end{array}$} \\
\hline
\end{tabular}

sepsis, bone or nerve injury, encephalopathy, or death. These analyses were performed with time expressed both as a continuous variable and as a categorical variable in 3-hour increments.

Generalized linear models for binary outcome with log-link function were used to estimate relative risks with 95\% confidence intervals for the association of latent phase duration (expressed as a continuous variable in hours) with obstetric outcomes. The CochranArmitage or exact test ${ }^{9}$ for trend was used to assess whether the frequency of obstetric outcomes changed as a function of the duration of the latent phase in

\section{TABLE 2 \\ Proportion of women no longer in latent phase after initiation of labor induction}

\begin{tabular}{llll}
$\begin{array}{l}\text { Latent } \\
\text { phase, } \mathrm{h}\end{array}$ & $\mathrm{N}$ & \multicolumn{1}{l}{$\%$} & \multicolumn{1}{l}{$\begin{array}{l}\text { Cumulative } \\
\%\end{array}$} \\
\hline $0-2.9$ & 3523 & 33.0 & 33.0 \\
\hline $3-5.9$ & 3470 & 32.5 & 65.5 \\
\hline $6-8.9$ & 1997 & 18.7 & 84.2 \\
\hline $9-11.9$ & 921 & 8.6 & 92.8 \\
\hline $12-14.9$ & 380 & 3.6 & 96.4 \\
\hline $15-17.9$ & 192 & 1.8 & 98.2 \\
\hline$\geq 18$ & 194 & 1.8 & 100.0 \\
\hline
\end{tabular}

Women at least $5 \mathrm{~cm}$ (or who had cesarean within given time interval) after cervical ripening had been completed, oxytocin had begun, and rupture of membranes (either spontaneously or artificially) had occurred.

Grobman et al. Defining failed induction of labor. Am J Obstet Gynecol 2018.

3-hour increments. A relative risk for each 3-hour interval was estimated with the generalized linear model utilizing the midpoint of the time interval as the value for the independent variable in the equation. The proportion of cesareans that occurred in the latent phase, in the active phase, and in the second stage, with the primary indications of nonreassuring fetal status (which included nonreassuring fetal heart tracing, cord prolapse, or abruption) or dystocia, was calculated for each 3-hour time interval as well.

To assess the robustness of our findings, several sensitivity analyses were performed. In one sensitivity analysis, the results of the analysis were reestimated after the number of missing time values was reduced. In this analysis, the latent phase starting time was assigned according to the time at ROM or oxytocin, when only 1 of those times was available. In further sensitivity analyses, generalized linear models with the log-link function, with time as a continuous independent variable, were rerun after adjusting for each of the following 3 factors: cervical ripening used (yes/no), elective induction (yes/no), or premature ROM (PROM) (yes/no). Adjustment in these models included the main effect of the factor as well as the interaction of the factor with time. Software (SAS, Version
9.4; SAS Institute Inc, Cary, NC) was used for the analyses. All tests were 2sided and $P<.05$ was used to define statistical significance with no adjustment for multiple comparisons.

\section{Results}

A total of 10,677 women met inclusion criteria and were available for analysis (Figure), 1725 (16.2\%) of whom underwent induction for PROM and 5582 (52.3\%) of whom underwent cervical ripening (Table 1). For women who did not present with PROM, the median duration between initiation of oxytocin and ROM was 215 minutes (interquartile range [IQR] 75-418 minutes) for women who underwent cervical ripening and 180 minutes (IQR 65-332 minutes) for women who did not undergo cervical ripening. The median duration from having had both oxytocin started and ROM (defined in this analysis as the start of the latent phase) to active labor (or cesarean delivery if active labor was not reached) was 262 minutes (IQR 141-435 minutes). By 6 hours almost two thirds of women had progressed from the start of the latent phase to active labor, and in the vast majority (96.4\%) of women, the active phase had been reached by 15 hours (Table 2).

The longer the duration of a woman's latent phase, the greater her chance of ultimately undergoing a cesarean delivery $(P<.001$ for time both as a continuous and categorical independent variable) (Table 3). Nevertheless, $>40 \%$ of women whose latent phase lasted for $\geq 18$ hours delivered vaginally. The indications for cesarean, stratified by phase and stage of labor, are presented in Table 4. As is illustrated, the majority of the cesareans at any of the time intervals-and in particular at the earlier time intervals that were $<15$ hours-were not performed in the latent phase. Several maternal morbidities (ie, chorioamnionitis, postpartum hemorrhage, and blood transfusion) also increased in frequency as the length of the latent phase increased.

Conversely, the frequency of most neonatal adverse outcomes did not differ as a function of time. Specifically, there was no statistical increase in the frequency of the primary neonatal adverse 


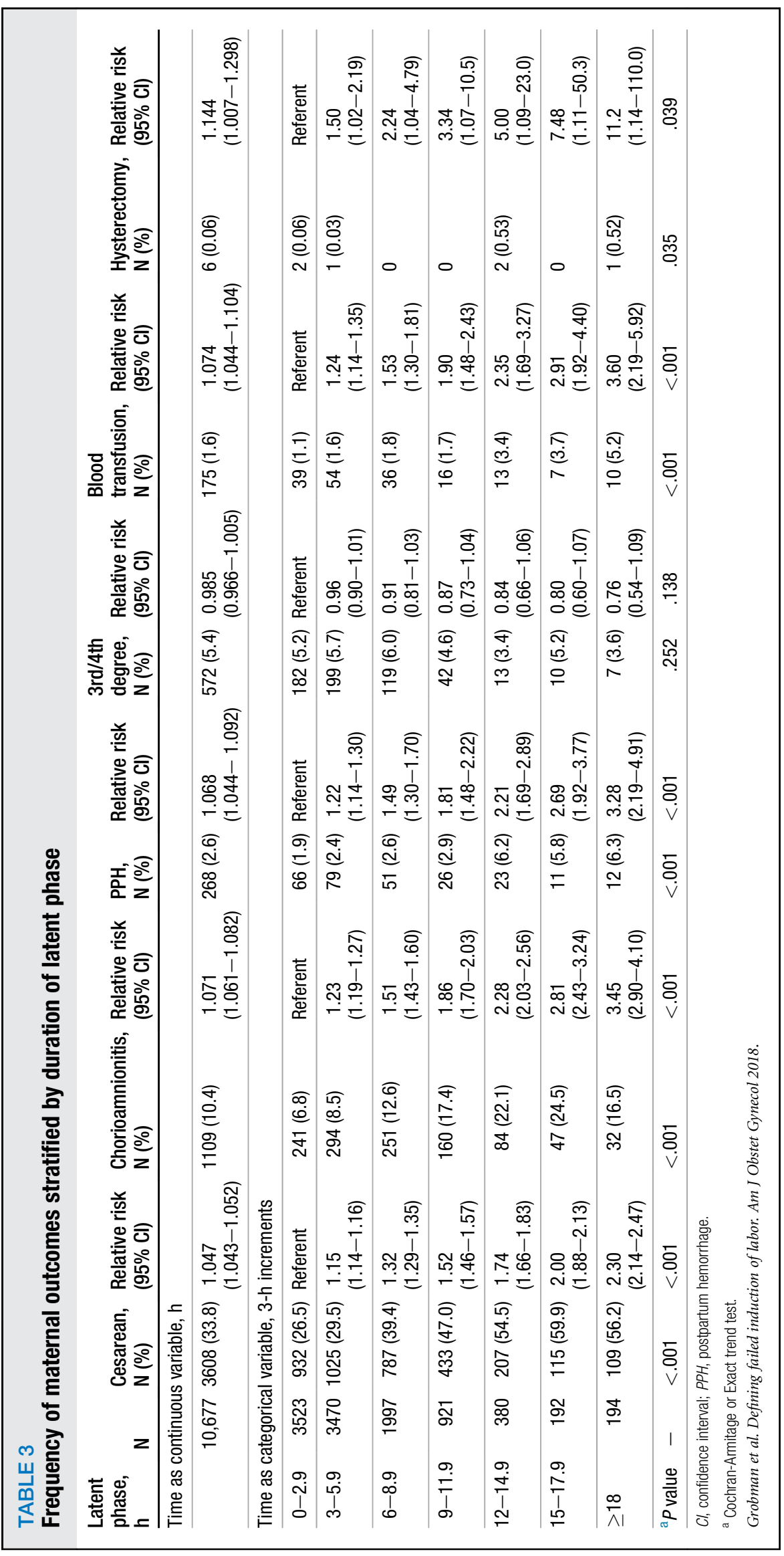

outcome, or of outcomes such as low Apgar score, acidemia, or shoulder dystocia. The frequency of neonatal intensive care unit (NICU) admission increased with duration of the latent phase (Table 5).

Lastly, for sensitivity analyses, we examined whether results differed after the number of missing values for latent phase duration were reduced (leaving only 746 latent phase durations missing), or after adjustment for whether cervical ripening had been used, the induction was undertaken without a medical indication (ie, an elective induction), or PROM had occurred. In all sensitivity analyses, the associations between duration of the latent phase and outcomes remained similar to those of the primary analysis (data not shown).

\section{Comment}

This study described several aspects of the latent phase in the setting of labor induction that may be helpful when considering recommendations regarding management of labor. First, a majority of women (ie, $>96 \%$ ) will enter the active phase within 15 hours of the completion of cervical ripening (if any is needed), the initiation of oxytocin, and ROM. The women who do are more likely than not to have a vaginal delivery and be free of maternal and perinatal morbidity. These patterns were extant regardless of whether the induction was without medical indication, was after PROM, or was after cervical ripening. Also, there is no one time at which complications suddenly arise, although there is an incremental increase in the frequency of several maternal complications, and of NICU admission, as time progresses.

These findings extend the findings of other investigators who have performed similar analyses. ${ }^{3-6}$ Studies such as those of Chelmow et $\mathrm{al}^{10}$ and Maghoma and Buchmann, ${ }^{11}$ for example, demonstrated that prolonged latent phases were associated with more frequent maternal and neonatal complications, although these studies neither were restricted to labor induction nor used a single consensus definition of "prolonged." Rouse et $\mathrm{al}^{3}$ performed the first study that used a similar approach to that of 
TABLE 4

Frequency of indications for cesarean delivery, stratified by phase and stage of labor

\begin{tabular}{|c|c|c|c|c|c|c|}
\hline \multirow[b]{2}{*}{ Latent phase, $\mathrm{h}$} & \multicolumn{2}{|c|}{ Latent phase } & \multicolumn{2}{|c|}{ Active phase } & \multicolumn{2}{|c|}{ Second stage } \\
\hline & NRFS & Dystocia & NRFS & Dystocia & NRFS & Dystocia \\
\hline $0-2.9[\mathrm{~N}=932]$ & $144(15.5)$ & $41(4.4)$ & $178(19.1)$ & $369(40.1)$ & $50(5.4)$ & $148(15.9)$ \\
\hline $3-5.9[\mathrm{~N}=1025]$ & $114(11.1)$ & $144(14.0)$ & $129(12.6)$ & 385 (37.6) & $46(4.5)$ & $206(20.1)$ \\
\hline $6-8.9[\mathrm{~N}=787]$ & $77(9.8)$ & $234(29.7)$ & $74(9.4)$ & $271(34.4)$ & $19(2.4)$ & 109 (13.9) \\
\hline $9-11.9[\mathrm{~N}=433]$ & $39(9.0)$ & $139(32.1)$ & $38(8.8)$ & $151(34.9)$ & $9(2.1)$ & $57(13.2)$ \\
\hline $12-14.9[\mathrm{~N}=207]$ & $25(12.1)$ & $71(34.3)$ & $16(7.7)$ & $65(31.4)$ & $4(1.9)$ & 24 (11.6) \\
\hline $15-17.9[\mathrm{~N}=115]$ & $9(7.8)$ & $55(47.8)$ & $9(7.8)$ & $31(27.0)$ & $3(2.6)$ & $8(7.0)$ \\
\hline$\geq 18[N=109]$ & $9(8.3)$ & $48(44.0)$ & $5(4.6)$ & $33(30.3)$ & $2(1.8)$ & $12(11.0)$ \\
\hline
\end{tabular}

Data presented as $\mathrm{N}(\%)$.

NRFS, nonreassuring fetal status.

Grobman et al. Defining failed induction of labor. Am J Obstet Gynecol 2018.

the present analysis to try to determine the association between duration of the latent phase and obstetric complications in the setting of labor induction. Their study included 509 women of mixed parity and demonstrated that "continued labor induction allowed some women to have vaginal deliveries," that chorioamnionitis rose with longer times of the latent phase, and that major maternal and perinatal complications were uncommon. They did not, however, have sufficient sample size to compare more uncommon neonatal complications, such as umbilical artery $\mathrm{pH}<7.0$, according to latent phase duration or reliably estimate the outcomes after $\geq 12$ hours of the latent phase.

Subsequent analyses by Simon and Grobman $^{4}(\mathrm{n}=397)$ and Rouse et $\mathrm{al}^{5}$ $(\mathrm{n}=1347)$ included more nulliparous women with latent phase durations $>12$ hours and concluded that even after 12 hours in the latent phase, vaginal delivery occurred with reasonable frequency and complications remained uncommon. For example, in the analysis by Simon and Grobman, ${ }^{4}$ 67\% of women who had a latent phase of 12-18 hours after the completion of any cervical ripening, oxytocin initiation, and ROM had a vaginal delivery without a discernible increase in perinatal complications. In a recent analysis of data from 9763 nulliparous women in the Consortium of Safe Labor study, in which $6 \mathrm{~cm}$ was used to define the end of latent labor, admission to the NICU (but not mechanical ventilation or sepsis) was statistically more frequent $(8.7 \%$ at 12 hours vs $6.7 \%$ at 9 hours) once 12 hours of the latent phase had been reached. ${ }^{6}$ While that study used $6 \mathrm{~cm}$ to define the end of the latent phase, the results are similar to our study (in which $5 \mathrm{~cm}$ was used as the terminal dilation for the latent phase).

There are several strengths of this study, including the size of the population, the quality of the data (which were abstracted by trained research personnel from each chart), and the diversity of a nationwide cohort. Women were included with a variety of indications for labor induction and with differing needs for cervical ripening, and the associations observed remained present even after taking these factors into account. Conversely, factors such as maternal age or body mass index were not adjusted for, given that these may not be mere covariates but causally related to both exposure and outcome. ${ }^{12}$

Despite these strengths, its limitations should be acknowledged. Because of its observational nature, the associations observed cannot be known to imply causality. Even with good data quality and control processes, particular types of data (eg, times for multiple events throughout labor) may be missing in the chart and thus not able to be abstracted.
If this missingness is not random, but related systematically to the outcome and exposure, bias may be introduced. However, in a sensitivity analysis in which the number of missing times was reduced by $>75 \%$, the results remained unchanged. Also, these findings are for nulliparous women and cannot be generalized to parous women. Nevertheless, we believe nulliparous women are the population most in need of study, given their much greater chance of prolonged labor and cesarean delivery. Because this study was concerned with the length of the latent phase during induction, it provides no insight into the latent phase during spontaneous labor. Although this study occurred at many institutions, most were academic centers with training programs, and thus generalizability to community hospitals cannot be certain. Yet, it is not evident why the presence of associations between duration of a phase of labor and obstetric outcomes should differ based on community or academic setting. And, one could consider the many institutions and the lack of a single protocol for induction or labor management as a strength of the study, as these characteristics increase the applicability of the findings to other institutions, which similarly lack a single standard for all aspects of labor management. Other studies of labor standards, such as those from the Consortium of Safe Labor, also have 


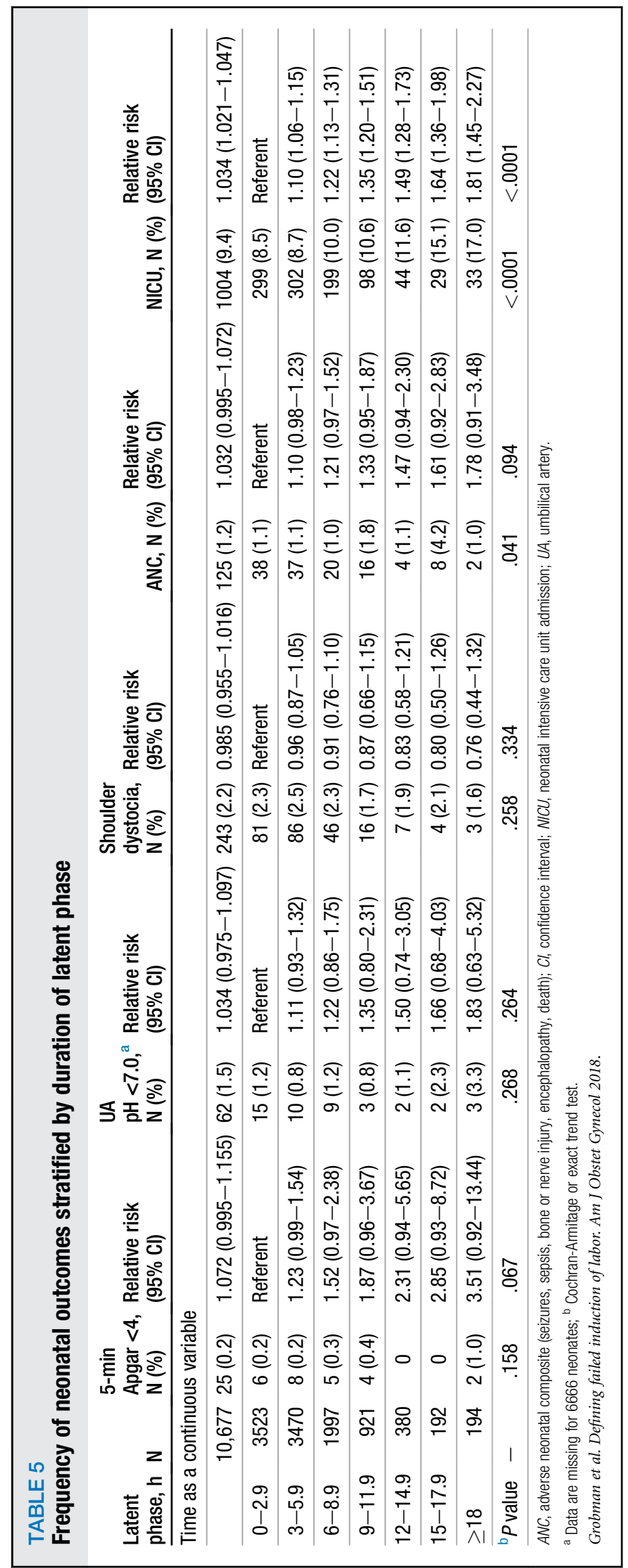

benefited from analysis of labor management as it occurs in actual clinical settings and not after imposition of a standard study protocol. ${ }^{13}$ Nevertheless, we cannot know with certainty whether these findings are generalizable to all health care settings.

This study presents one, but certainly not the only approach, to assessing the extent to which the latent phase should continue, in the absence of acute indication for delivery, during labor induction. Other approaches, for example, include determination of inflection points on labor curves as well as categorizing abnormality based on population-level percentiles. ${ }^{13-16}$ This presently applied approach, however, has several advantages, including that it standardizes the duration as a function of several aspects of management to establish a common "clock" and directly assesses the relationship between duration and obstetric outcome. Indeed, some investigators have stressed the importance of determining labor definitions and utility of different management approaches in the context of maternal and neonatal outcomes. ${ }^{17,18}$ Regardless of the specific approach, a standard for the minimum duration of the latent phase that should be employed in the setting of labor induction is sorely needed. Obstetric providers are routinely faced with the question, for women who have not entered the active phase, of whether the benefits of allowing an induction to continue outweigh the risks. Lacking a standard, interpatient and interinstitutional variability result. As our data demonstrate, even though cesarean for dystocia or failed induction was not frequent, it not only was cited as an indication but occurred at a variety of durations throughout the latent phase.

Converting these data into a discrete clinical recommendation is challenging given there is no single time interval at which the complications suddenly arise, at which the marginal increase in the frequency of complications dwarfs the marginal increase during antecedent intervals, or at which there is no longer a balance of benefit (eg, avoidance of additional cesareans) with risk. 
However, given that the vast majority of women will progress to the active phase by 15 hours, that most women who do will progress to a vaginal delivery, and that relatively few will have adverse outcomes, we believe that our results are consistent with prior recommendations. $^{3-5,7}$ Specifically, among women undergoing labor induction, when maternal and fetal maternal and fetal conditions permit, cesarean delivery should not be undertaken in the latent phase prior to at least 15 hours after ROM has occurred and oxytocin has been started. The decision to continue labor beyond this point should be individualized, and may take into account factors such as other evidence of labor progress.

The advantages of such an approach are illustrated by 1 study that demonstrated that introduction of a protocol that specified a minimum amount of time before a failed induction was diagnosed (in that case, 12 hours after ripening, oxytocin, and ROM) was associated with a significantly lower frequency of cesarean and no greater frequency of adverse outcomes. ${ }^{19}$ And, based on our data, the public health ramifications of adherence to a standard definition for "failed induction" can be estimated. If induction were to be designated as "failed" if a woman continued to be in the latent phase at 15 as opposed to 6 hours after the initiation of oxytocin and ROM, approximately 70,000 additional cesarean deliveries could be avoided among the 400,000 nulliparous women who undergo induction in the United States every year.

\section{Acknowledgment}

The authors thank Cynthia Milluzzi, RN, and Joan Moss, RNC, MSN, for protocol development and coordination between clinical research centers; and Elizabeth Thom, PhD, Madeline M. Rice, PhD, Brian M. Mercer, MD, and Catherine Y. Spong, MD, for protocol development and oversight.

In addition to the authors, other members of the Eunice Kennedy Shriver National Institute of Child Health and Human Development Maternal-Fetal Medicine Units Network are as follows:

Northwestern University, Chicago, IL-G. Mallett, M. Ramos-Brinson, A. Roy, L. Stein, P.
Campbell, C. Collins, N. Jackson, M. Dinsmoor (NorthShore University HealthSystem), J. Senka (NorthShore University HealthSystem), K. Paychek (NorthShore University HealthSystem), A. Peaceman.

Columbia University, New York, NY-M. Talucci, M. Zylfijaj, Z. Reid (Drexel University), R. Leed (Drexel University), J. Benson (Christiana Hospital), S. Forester (Christiana Hospital), C. Kitto (Christiana Hospital), S. Davis (St Peter's University Hospital), M. Falk (St Peter's University Hospital), C. Perez (St Peter's University Hospital).

University of Utah Health Sciences Center, Salt Lake City, UT-K. Hill, A. Sowles, J. Postma (LDS Hospital), S. Alexander (LDS Hospital), G. Andersen (LDS Hospital), V. Scott (McKayDee), V. Morby (McKay-Dee), K. Jolley (Utah Valley Regional Medical Center), J. Miller (Utah Valley Regional Medical Center), B. Berg (Utah Valley Regional Medical Center).

University of North Carolina at Chapel Hill, Chapel Hill, NC-K. Dorman, J. Mitchell, E. Kaluta, K. Clark (WakeMed), K. Spicer (WakeMed), S. Timlin (Rex), K. Wilson (Rex).

University of Texas Southwestern Medical Center, Dallas, TX-L. Moseley, M. Santillan, J. Price, K. Buentipo, V. Bludau, T. Thomas, L. Fay, C. Melton, J. Kingsbery, R. Benezue.

University of Pittsburgh, Pittsburgh, PA-H. Simhan, M. Bickus, D. Fischer, T. Kamon (deceased), D. DeAngelis.

Case Western Reserve UniversityMetroHealth Medical Center, Cleveland, $\mathrm{OH}-\mathrm{B}$. Mercer, C. Milluzzi, W. Dalton, T. Dotson, P. McDonald, C. Brezine, A. McGrail.

Ohio State University, Columbus, $\mathrm{OH}-\mathrm{C}$. Latimer, L. Guzzo (St Ann's), F. Johnson, L. Gerwig (St Ann's), S. Fyffe, D. Loux (St Ann's), S. Frantz, D. Cline, S. Wylie, J. lams.

University of Alabama at Birmingham, Birmingham, AL-M. Wallace, A. Northen, J. Grant, C. Colquitt, D. Rouse, W. Andrews.

University of Texas Medical Branch, Galveston, TX-J. Moss, A. Salazar, A. Acosta, G. Hankins.

Wayne State University, Detroit, Ml-N. Hauff, L. Palmer, P. Lockhart, D. Driscoll, L. Wynn, C. Sudz, D. Dengate, C. Girard, S. Field.

Brown University, Providence, RI-P. Breault, F. Smith, N. Annunziata, D. Allard, J. Silva, M. Gamage, J. Hunt, J. Tillinghast, N. Corcoran, M. Jimenez.

University of Texas Health Science Center at Houston-Children's Memorial Hermann Hospital, Houston, TX-F. Ortiz, P. Givens, B. Rech, C. Moran, M. Hutchinson, Z. Spears, C. Carreno, B. Heaps, G. Zamora.

Oregon Health and Science University, Portland, OR-J. Seguin, M. Rincon, J. Snyder, C. Farrar, E. Lairson, C. Bonino, W. Smith (Kaiser Permanente), K. Beach (Kaiser Permanente), S. Van Dyke (Kaiser Permanente), S. Butcher (Kaiser Permanente).

George Washington University Biostatistics Center, Washington, DC-E. Thom, M. Rice, Y. Zhao, P. McGee, V. Momirova, R. Palugod, B. Reamer, M. Larsen.
Eunice Kennedy Shriver National Institute of Child Health and Human Development, Bethesda, MD-C. Spong, S. Tolivaisa.

Maternal-Fetal Medicine Units Network Steering Committee Chair (Medical University of South Carolina, Charleston, SC)-J. P. Van Dorsten, MD.

\section{References}

1. Martin JA, Hamilton BE, Osterman MJ, Curtin SC, Mathews TJ. Births: final data for 2013. Natl Vital Stat Rep 2015;64:1-65.

2. Baños N, Migliorelli F, Posadas E, Ferreri J, Palacio M. Definition of failed induction of labor and its predictive factors: two unsolved issues of an everyday clinical situation. Fetal Diagn Ther 2015;38:161-9.

3. Rouse DJ, Owen J, Hauth JC. Criteria for failed labor induction: prospective evaluation of a standardized protocol. Obstet Gynecol 2000;96:671-7.

4. Simon CE, Grobman WA. When has an induction failed? Obstet Gynecol 2005;105: 705-9.

5. Rouse DJ, Weiner SJ, Bloom SL, et al. Failed labor induction: toward an objective diagnosis. Obstet Gynecol 2011;117:267-72.

6. Kawakita $T$, Reddy UM, labal SN, et al. Duration of oxytocin and rupture of the membranes before diagnosing a failed induction of labor. Obstet Gynecol 2016;128:373-80.

7. Caughey AB, Cahill AG, Guise JM, Rouse DJ. Safe prevention of the primary cesarean delivery. Am J Obstet Gynecol 2014;210:179-93.

8. Bailit JL, Grobman WA, Rice MM, et al. Riskadjusted models for adverse obstetric outcomes and variation in risk-adjusted outcomes across hospitals. Am J Obstet Gynecol 2013;209:446. e1-30.

9. Agresti A. A survey of exact inference for contingency tables. Stat Sci 1992;7:131-77.

10. Chelmow D, Kilpatrick SJ, Laros RK Jr. Maternal and neonatal outcomes after prolonged latent phase. Obstet Gynecol 1993;81: 486-91.

11. Maghoma J, Buchmann EJ. Maternal and fetal risks associated with prolonged latent phase of labor. J Obstet Gynaecol 2002;22: 16-9.

12. Ananth CV, Schisterman EF. Confounding, causality, and confusion: the role of intermediate variables in interpreting observational studies in obstetrics. Am J Obstet Gynecol 2017;217: 167-75.

13. Zhang J, Landy $H$, Branch DW, et al. Contemporary patterns of spontaneous labor with normal neonatal outcomes. Am J Obstet Gynecol 2010;116:1281-7.

14. Friedman EA. The graphic analysis of labor. Am J Obstet Gynecol 1954;68:1568-75.

15. Friedman EA. Primigravid labor: a graphicostatistical analysis. Obstet Gynecol 1955;6: 567-89.

16. Zhang J, Troendle J, Yancey MK. Reassessing the labor curve in nulliparous women. Am J Obstet Gynecol 2002;187:824-8. 
17. Hanley GE, Munro S, Greyson D, et al. Diagnosing onset of labor: a systematic review of definitions in the research literature. BMC Pregnancy Childbirth 2016;16:71.

18. Lavender T, Hart A, Smyth R. Effect of partogram use on outcomes for women in spontaneous labor at term. Cochrane Database Syst Rev 2013;7:CD005461.

19. Rhinehart-Ventura J, Eppes C, SangiHaghpeykar $\mathrm{H}$, et al. Evaluation of outcomes after implementation of an induction-of-labor protocol. Am J Obstet Gynecol 2014;211:301. e1-7.

\section{Author and article information}

From the Departments of Obstetrics and Gynecology of Northwestern University, Chicago, IL (Dr Grobman);
MetroHealth Medical Center-Case Western Reserve University, Cleveland, $\mathrm{OH}$ (Dr Bailit); George Washington University Biostatistics Center, Washington, DC (Dr Lai); Eunice Kennedy Shriver National Institute of Child Health and Human Development, Bethesda, MD (Dr Reddy); Columbia University, New York, NY (Dr Wapner); University of Utah Health Sciences Center, Salt Lake City, UT (Dr Varner); University of North Carolina at Chapel Hill, Chapel Hill, NC (Dr Thorp); University of Texas Southwestern Medical Center, Dallas, TX (Dr Leveno); University of Pittsburgh, Pittsburgh, PA (Dr Caritis); Ohio State University, Columbus, $\mathrm{OH}$ (Dr Prasad); University of Alabama at Birmingham, Birmingham, AL (Dr Tita); University of Texas Medical Branch, Galveston, TX (Dr Saade); Wayne State University, Detroit, MI (Dr Sorokin); Brown University, Providence, RI (Dr Rouse); University of Texas Health Science Center at Houston-Children's Memorial Hermann
Hospital, Houston, TX (Dr Blackwell); and Oregon Health and Science University, Portland, OR (Dr Tolosa).

Received Oct. 2, 2017; revised Oct. 27, 2017; accepted Nov. 6, 2017.

The project described was supported by grants from the Eunice Kennedy Shriver National Institute of Child Health and Human Development (HD21410, HD27869, HD27915, HD27917, HD34116, HD34208, HD36801, HD40500, HD40512, HD40544, HD40545, HD40560, HD40485, HD53097, HD53118) and the National Center for Research Resources (UL1 RR024989; 5UL1 RR025764). Comments and views of the authors do not necessarily represent views of the National Institutes of Health.

The authors report no conflict of interest.

Corresponding author: William A. Grobman, MD, MBA.w-grobman@northwestern.edu 